\section{Uso de los simuladores en odontología pospandemia}

\section{Use of simulators in post-pandemic dentistry}

\section{Resumen}

La pandemia ocasionada por el virus de la COVID-19 determinó que se tomaran medidas sanitarias que afectaron el normal desarrollo, no solo de las actividades laborales sino también de las académicas. La prohibición de la presencialidad en las universidades, específicamente en las facultades de odontología donde la actividad clínica en los últimos tres años de estudio es fundamental para el desarrollo de las competencias procedimentales y actitudinales quedaron totalmente suspendidas. El presente artículo tiene como objetivo revisar el uso de los simuladores pospandemia, como una tecnología que permita, gracias a sus alcances, suplir la presencia de los pacientes. Para ello se realizó una búsqueda de artículos científicos en las siguientes bases de datos: Google académico, MEDLINE/PubMed, SciELO, Scopus y LILACS, durante los años 2020-2021; los artículos recolectados estuvieron relacionados con la importancia del uso de simuladores en el desarrollo de las competencias requeridas. De acuerdo con los resultados obtenidos se pudo determinar que el uso de esta tecnología permitió básicamente desarrollar habilidades, destreza y confianza en los estudiantes sin tener que poner en riesgo la salud e integridad física del paciente, con la posibilidad de evaluar y repetir los procedimientos realizados indeterminadamente.

Palabras clave: Simulación; Educación superior; COVID-19 (fuente: DeCS BIREME).

\section{Artículo de Revisión}

\author{
Walter Gallo-Zapata ${ }^{1, a}$, Hans Contreras-Pulache ${ }^{2, b}$, Ana \\ Maria Diaz-Soriano ${ }^{1,0}$ \\ ${ }^{1}$ Universidad Nacional Mayor de San Marcos, Facultad \\ de Odontología, Lima, Perú. \\ ${ }^{2}$ Universidad Norbert Wiener, Lima, Perú. \\ a Especialista en Rehabilitación Oral. \\ ${ }^{\mathrm{b}}$ Magíster en Salud Publica. \\ ${ }^{c}$ Magíster en Estomatología.

\section{Correspondencia:} \\ Walter Gallo-Zapata: wgalloz@unmsm.edu.pe \\ Jr. Juan Voto Bernales 294 Santa Catalina Lima-13 \\ ORCID: 0000-0002-0717-8915
}

\section{Coautores:}

Hans Contreras-Pulache: hcontrerasp@unmsm.edu.pe ORCID: 0000-0003-2450-9349

Ana Maria Diaz-Soriano: adiazs@unmsm.edu.pe

ORCID: 0000-0002-3760-0902

\section{Editora:}

Rosse Mary Falcón-Antenucci

Universidad Inca Garcilaso de la Vega.

Conflicto de intereses: los autores declaran no tener conflictos de interés.

Fuente de financiamiento: autofinanciado.

Recibido: 23/09/21

Aceptado: 27/10/21

Publicado: $21 / 01 / 22$ 
that the use of this technology, basically allows the development of skills, dexterity and confidence in the students without having to put the health and physical integrity of the patient at risk, with the possibility of evaluating and repeating the procedures performed indefinitely.

Keywords: Simulator; Higher education; COVID-19 (source: MeSH NLM).

\section{Introducción}

El mayor impacto visible a corto plazo generado por la COVID-19, según el reporte de la UNESCO, fue el cierre de las universidades y por ende la suspensión de las actividades presenciales, debiéndose optar por alternativas que permitan la continuidad de las actividades docentes ${ }^{1}$. Es importante resaltar que estos fueron los efectos inmediatos, pero no a mediano o largo plazo; el escenario actual que viven los alumnos de pregrado es totalmente nuevo, sin idea alguna de cuánto tiempo va a durar esta situación. En el profesorado el mayor impacto es la expectativa de continuidad bajo la modalidad virtual, siendo más vulnerable el personal no docente ante la posibilidad de una reducción de puestos de trabajo ${ }^{2}$.

La pandemia ocasionada por la COVID-19 ha afectado doblemente el proceso educativo en las facultades de odontología, porque al suspenderse la presencialidad de las actividades académicas; se ha optado por la enseñanza online. Además, se suspendió la atención a los pacientes, por considerarse que los procedimientos clínicos generan un alto riesgo de contagio, debido a los fluidos del paciente y los equipos ${ }^{3}$. El uso de la simulación en odontología es una gran alternativa que puede permitir la continuidad de las actividades preclínicas e inclusive clínicas, bajo estrictas medidas de bioseguridad, esto permitirá el desarrollo de las competencias requeridas en la formación de los alumnos, tales como: destreza y habilidad, previa a la atención clínica de pacientes ${ }^{4}$.

Para hacer frente a la pandemia, las facultades de odontología requieren de la implementación de medidas que garanticen el desarrollo de las actividades presenciales mediante el uso de nuevas tecnologías que reduzcan el riesgo de contagio del SARS-CoV-2 y aseguren la calidad de la educación ${ }^{5}$.

Las dificultades en la enseñanza generadas por la COVID-19 han dado origen a la modernización de los enfoques pedagógicos con el uso de tecnologías digitales, las mismas que muchas veces estuvieron subestimadas en el proceso educativo y en los servicios de salud oral, como es el caso de la Teleodontología, que adquiere un gran protagonismo en el diagnóstico y prevención ${ }^{6,7}$, así como la simulación en el entrenamiento de los alumnos.

En tiempos de pospandemia el uso de la simulación se presenta como la mejor opción, al no exponer a los pacientes, ni a los alumnos durante el desarrollo de las prácticas clínicas, incrementando la autoconfianza, reduciendo errores y disminuyendo el riesgo de contagio. La implementación de esta tecnología implica recursos económicos, materiales y un programa regular de entrenamiento ${ }^{8}$.
Es importante resaltar el tema de la seguridad de los pacientes; en un informe publicado el año 2000 titulado "Errar es humano" se muestra que aproximadamente 98 000 pacientes murieron a causa de errores médicos en Estados Unidos (EUA), superando a las muertes causadas por enfermedades, accidentes de tránsito, enfermedades oncológicas o SIDA. Las nuevas exigencias de la cultura de seguridad, éticamente ya no son toleradas en las prácticas clínicas, debido a que se pone en riesgo la vida de los pacientes, siendo el uso de la simulación previa a la atención clínica, una opción segura para el aprendizaje de los alumnos ${ }^{9}$. La presente revisión de la literatura tiene como objetivo mostrar la importancia y beneficios de incorporar el uso de simuladores en el proceso de desarrollo de las competencias procedimentales preclínicas y clínicas (a futuro) de las facultades de odontología.

\section{Métodos}

Se realizó la búsqueda bibliográfica durante los años 2020-2021, en las bases de datos: Google académico, MEDLINE/PubMed, SciELO, Scopus y LILACS; usando palabras clave en los idiomas español e inglés.

Pandemia COVID-19. Desde el inicio de la pandemia en Wuhan (China), el coronavirus se propagó rápidamente en 114 países. La Organización Mundial de la Salud (OMS) ${ }^{10}$ declaró pandemia a la COVID-19 el 11 de marzo del 2020 y en setiembre 2021 estimó entre 6,8 a 9 millones de fallecidos.

Los coronavirus son los virus de ARN más grande, variando su tamaño de 65 a $125 \mathrm{~nm}$ de diámetro, el Sars-CoV-2 es el séptimo virus conocido desde 1960 y pertenece al género beta coronavirus, su tasa de mortalidad es inferior $(2,3 \%)$ a la del SRAS $(9,5 \%)$ y al del MERS $(34,4 \%)^{11}$. El mecanismo de transmisión es por medio del contacto de las gotas de los fluidos al hablar, estornudar o toser y aerosoles generados durante la atención odontológica, su origen es nasofaríngeo u orofaríngeo. Estas gotas pueden quedar suspendidas en el aire y producir transmisión a larga distancia, se han encontrado cepas del virus en saliva después de 29 días de la infección ${ }^{12}$.

El impacto generado por la COVID-19 en la salud de las personas que se ven amenazadas mortalmente, es que toda actividad queda bloqueada. En las universidades todo el ecosistema de enseñanza tuvo que implementar la modalidad virtual (online), siendo necesario un nuevo diseño integral, un cambio del proceso de evaluación y metodologías que permitieron un elevado nivel de participación y satisfacción de los alumnos. Los éxitos logrados en la Universidad de Salamanca fue producto del empleo de metodologías activas y de la incorporación 
de tecnologías educativas empleadas durante la presencialidad que permitió la conversión a un enfoque online o mixto, no obteniéndose la reducción de los niveles de satisfacción en comparación con cursos anteriores ${ }^{13}$.

Los cambios provocados por la pandemia en la educación fueron intempestivos. Las plataformas tecnológicas, docentes, y alumnos no estaban preparados para ello, no hubo el tiempo necesario para una planificación en las universidades, ni de la disposición de los recursos y dispositivos en los hogares, generando una situación de estrés que se agudizó durante el proceso de evaluación en los cursos online, porque esta se realizaba presencialmente, una de las formas que se propusieron fue optar por una evaluación continua, reduciendo al máximo las pruebas finales. Tres son los escenarios propuestos: la presencialidad, online y el modelo híbrido. Se apuesta a que la flexibilidad, planificación y la organización permita un buen uso de las tecnologías ${ }^{13,14}$.

El año académico 2019-2020 fue sorprendido por la presencia del SARS-CoV-2, y adecuándose al periodo de emergencia, las clases presenciales fueron cambiadas por clases virtuales, siendo detectadas ciertas deficiencias con relación a las habilidades digitales y dificultades del acceso al internet, tanto de los alumnos como de los docentes, agudizando las brechas ya existentes.

Las videoconferencias y el PowerPoint fueron las herramientas más usadas. En el mes de junio se presentaron complicaciones debido al proceso de evaluación; la propuesta de la evaluación continua no funcionó generando descontento a todo nivel, por lo que se requirió un cambio profundo en el proceso de evaluación. A inicios del 2020 la amenaza del virus se mantuvo latente, se ha replanteado la propuesta de algunas universidades en lo que se ha denominado "presencialidad segura", "docencia híbrida", sin mayores resultados, es conveniente el abordaje de nuevos modelos educativos ${ }^{15}$.

Las universidades deben tener un plan de contingencia para futuras eventualidades, el manejo de las tecnologías de la información y comunicación requiere fundamentalmente del entrenamiento de los profesores. Para encontrar soluciones, no es suficiente una notable inversión en tecnología de punta, sino que se requiere del esfuerzo fusionado, colaborativo en función de las necesidades del país, las instituciones y la universidad ${ }^{16}$. La modalidad online ${ }^{17}$ a la que se denominó "enseñanza remota de emergencia" evidenció un déficit estructural en las universidades. Las secuelas de esta pandemia en la educación, aún serían prematuro expresarlas, mientras no se realice un análisis y evaluación de la calidad de este modelo de enseñanza, la implementación requiere de tecnología, flexibilidad de tiempo y autosuficiencia del estudiante. Es evidente que el cierre de universidades, debido a la pandemia, abrió una gran brecha en lo que se refiere a la desigualdad de oportunidades entre las familias que tienen o no una situación socioeconómica favorable como para disponer de un equipamiento tecnológico y conectividad al internet, situación que se agrava considerando la necesidad del uso múltiple de cada miembro de la familia. Esta brecha puede ser causa de la interrupción o abandono del proceso educativo ${ }^{18}$.

$\mathrm{Al}$ ser afectada la actividad universitaria a nivel mundial, nuevas estrategias pedagógicas se implantaron en las facultades. En México se incrementó la capacitación y uso de la plataforma virtual Blackboard, así también, se tuvo que digitalizar todas las asignaturas ${ }^{19}$. La pandemia ha llevado a reevaluar las prácticas educativas estándar con el fin de definir lo que es realmente importante, siendo ello de gran valor para el docente y el discente. Las limitaciones y restricciones dispuestas permitieron buscar alternativas que permitan la transmisión del conocimiento, esto ha impulsado al docente a crear nuevos modelos educativos de la mano con las tecnologías digitales, que permitan un mayor acercamiento entre el profesor y el alumno superando distancias y tiempo ${ }^{20}$.

El trabajo en odontología se caracteriza por ser manual/ práctico, pero la presencia del SARS-CoV-2 bloqueó toda posibilidad del desarrollo de estas competencias procedimentales limitando la enseñanza al campo teórico. La presencia del virus en la saliva y su transmisión en el aerosol se convirtió en una amenaza para los dentistas, las disposiciones de los gobiernos para cerrar las universidades e imponer el distanciamiento social, redujo al 100\% las actividades clínicas en las facultades y esto condujo al colapso del sistema de educación en odontología ${ }^{21}$.

La enseñanza en odontología presenta grandes dificultades, que requieren de una vasta preparación del docente y del empleo de metodologías cuyo centro de aprendizaje sea el estudiante, que durante ese periodo deberá adquirir destrezas manuales y cognitivas necesarias para realizar sus tratamientos. El uso de las TICs permitió lograr un avance teórico, debiéndose postergar las actividades prácticas hasta un futuro retorno a la presencialidad.

La Federación Internacional de Escuelas y Facultades de Odontología (FIEFO) en el seminario online realizado en la Facultad de Odontología de la UIC Barcelona (junio 2020), frente a la crisis sanitaria, propuso la necesidad de un replanteamiento en los diferentes niveles educativos, considerando que la educación de pre y posgrado quedará afectada juntamente con el ejercicio profesional, por el riesgo que generan los aerosoles donde el virus puede sobrevivir varias horas. Esta situación permitirá la evolución de la enseñanza odontológica hacia el uso de tecnologías digitales que garanticen la continuidad de la enseñanza ${ }^{1}$.

Al cerrarse el curso académico 2019-2020, periodo en el que la universidad tuvo que migrar a una docencia a distancia, se logró impartir las materias y evaluar la mayor parte de las competencias, ya sea en forma urgente y sin una planificación, observándose una gran brecha tecnológica en lo que se refiere al acceso, uso y habilidades. La transformación digital de la universidad implica grandes cambios hacia un nuevo modelo educativo y operativo, no se trata de introducir tecnología para seguir haciendo lo mismo; es necesario una reingeniería de procesos siendo necesario el apoyo y liderazgo de los gobiernos universitarios. 
La reforma progresiva del Sistema Europeo de Educación Superior está orientada a que el principal protagonista sea el aprendizaje del estudiante, apostando no a la nota mínima global de la asignatura sino a la comprobación del logro de las competencias, es indudable que la docencia ya no será igual cuando esta pandemia se supere ${ }^{13}$.

El Plan Bolonia (1999) en las universidades buscó la unificación de criterios educativos en toda Europa dividiendo la enseñanza superior en tres niveles. Fomentando la formación integral y la madurez de la persona, donde la función del docente sea acompañar al alumno en su proceso de transformación. Todos los países europeos han hecho el esfuerzo de adaptarse a este modelo compartido, debido a la crisis sanitaria se tuvieron que adaptar al sistema de educación a distancia, debiendo para ello digitalizarse, dando lugar a un nuevo modelo híbrido que se apoya en la tecnología y sobre todo en la innovación pedagógica debiendo producirse transformaciones a nivel pedagógico y organizativo de tal forma que el aprendizaje remoto sea igual al presencial ${ }^{22}$.

Simuladores odontológicos. Las facultades de odontología actualmente enfrentan un gran desafío ocasionado por la COVID-19, debiendo para ello usar todos los recursos virtuales disponible que permitan la continuidad de sus actividades académicas. En la Facultad de Odontología de la Universidad de Marburg (Alemania), se paralizaron las actividades en la primavera del 2020, para posteriormente reiniciarlas completamente en el invierno del mismo año, bajo un nuevo concepto de higiene, aplicando pruebas rápidas de antígeno a sus pacientes, un régimen de higiene mejorada y el reclutamiento de pacientes. La implementación de la educación virtual y el uso de simuladores permitió dar continuidad a sus actividades en plena pandemia ${ }^{23}$.

La simulación es definida como "una técnica educativa que permite una actividad interactiva, $y$ a veces inmersiva, al recrear todo o parte de la experiencia clínica sin exponer a los pacientes a los riesgos asociados" ${ }^{24} \mathrm{y}$ tiene como objetivo fundamental recrear un evento clínico lo más real posible para la realización de un entrenamiento de una técnica de forma dinámica y segura ${ }^{25}$. El uso de la simulación como modalidad de enseñanza se ha incrementado, y como dice Pai, no es un entretenimiento ni una forma elegante de hacer algo nuevo, por lo tanto, debe ser considerada como una herramienta basada en los mismos principios educativos ${ }^{26}$. La simulación clínica permite el desarrollo de las competencias necesarias para la realización de los procedimientos clínicos en pacientes, posibilitando un mejor desempeño de los estudiantes, una mayor seguridad para los pacientes y un menor margen de error traducido en daño ${ }^{4}$.

La incorporación de la simulación al modelo pedagógico contribuirá en el proceso de aprendizaje mediante prácticas preclínicas antes de proceder a la atención de pacientes. La certificación de las habilidades y destrezas promoverán una cultura de seguridad del paciente ${ }^{27-29}$. En el ámbito de la salud se considera una representación controlada de la realidad ya sea reemplazándola o ampliándola, permitiendo el desarrollo de habilidades de pensamiento crítico sin afectar la seguridad del paciente ${ }^{27}$.

La implementación de la simulación requiere definir los objetivos de aprendizaje valorar su utilidad, el uso combinado de simulación de baja fidelidad y la simulación estandarizada permiten integrar la teoría con la práctica, asimismo el estudiante tendrá un primer acercamiento a un paciente real, y reflexionar sobre la importancia de generar una empatía con el paciente ${ }^{28}$.

El uso de simuladores forma parte del proceso del logro de las competencias procedimentales de los alumnos de odontología, y ha ido evolucionando desde el uso inicial de maxilares humanos con dientes, posteriormente maquetas de yeso con dientes de cadáveres, maquetas de acrílico, luego los fantomas y últimamente los simuladores virtuales hápticos, cuyo fin es lograr el desarrollo de habilidades cognitivas, motoras y actitudinales. Un estudio cuantitativo observacional, no experimental en la Universidad de San Sebastián (Chile) determinó la percepción en la utilidad de simuladores hápticos y se obtuvo una valoración positiva en su uso como herramienta de instrucción para el logro de las habilidades manuales ${ }^{29}$.

La educación no evolucionó al ritmo de las TICs, siendo necesario incentivar prácticas innovadoras en los procesos de enseñanza-aprendizaje con el apoyo de las TICs, generando nuevas estrategias para los sistemas de comunicación y distribución de materiales de aprendizaje. Para todo este cambio es importante la participación del docente quien decidirá las herramientas más adecuadas según los contenidos a impartir. La presencia de las TICs es indispensable en el área de salud y odontología ${ }^{30}$.

Se considera que la simulación es una técnica y no una tecnología, que permite generar experiencias reales en forma interactiva y guiada. Considerando sus aplicaciones en 11 dimensiones: objetivos y propósitos de la actividad de simulación, unidad de participación, nivel de experiencia de los participantes, dominio de la atención de la salud, disciplina profesional de los participantes, tipo de conocimientos, habilidades, actitudes o comportamientos abordados, edad del paciente simulado, tecnología aplicable o requerida, sitio de simulación, extensión de la participación directa y método de retroalimentación utilizado ${ }^{31}$.

Si bien el uso de la simulación clínica favorece el logro de las competencias requeridas para la realización de los procedimientos ante un escenario de pandemia, el fantomas se convierte en un portador asintomático, es por eso que se debe disponer de espacios que permitan el distanciamiento (según normas sanitarias) y un número apropiado de simuladores. Se debe tener en consideración la posibilidad de la presencia de portadores asintomáticos ${ }^{32}$. Los simuladores han demostrado ser una herramienta fiable para la evaluación del estudiante. Se hacen necesarios estudios que evalúen los resultados en los pacientes ${ }^{32,33}$.

La simulación clínica, según Corvetto, es producto de la influencia de cuatro fuerzas como se grafica en la Figura 
1, las cuales son producto de las variaciones que a través del tiempo se han producido en cada área, con un nuevo enfoque en que prima la seguridad e integridad del paciente $^{32-34}$.

La primera universidad que usó simulador en América Latina fue (en 1963) el Instituto Tecnológico y de Estudios Superiores de Monterrey, la forma más básica de simulación fue el uso de un diente natural colocado sobre una base de yeso en forma de maxilar, en la actualidad los más usados son los maniquís que poseen una cabeza artificial donde tiene insertados los maxilares, los mismos que se pueden acoplar a los sillones dentales creando un escenario clínico más real ${ }^{35}$.

Durante la ejecución de los procedimientos clínicos en pacientes los operadores están expuestos a una serie de movimientos y posiciones inadecuadas que pueden producir un deterioro en las articulaciones, tejidos óseos, musculares nerviosos, dependiendo de los tiempos de exposición a posturas forzadas, a ello se suma la carga mental que es afectada por agentes externos como el ruido de los equipos, la vibración del tránsito vehicular ${ }^{33}$. Durante el entrenamiento con los simuladores es posible formar, educar y corregir toda posición que ponga en peligro la salud del operador. La clasificación de los simuladores está centrada según el nivel de realismo de los prototipos, según su aplicación o su nivel de fidelidad ${ }^{35} \mathrm{y}$ de acuerdo con Ziv se dividen en cinco categorías $^{36}$ (Figura 2).

En simulación la fidelidad juega un rol importante para una tarea específica, no siempre la alta fidelidad es superior a la baja fidelidad, todo depende del tipo de tarea y el nivel del alumno, los criterios de evaluación en una simulación deben considerar la fidelidad, fiabilidad, validez, impacto en aprendizaje y viabilidad ${ }^{35,37}$. El término fidelidad en simulación está referido con la similitud a la realidad, lo cual no define la complejidad ni la tecnología, un paciente simulado tiene alta fidelidad, pero baja complejidad y escasa tecnología. Bajo estos conceptos los simuladores en odontología que utilizan maniquíes, ubicados en un sillón dental bien podría considerarse de alta fidelidad, pero de baja complejidad y tecnología. Bajo estos criterios en que se considera el grado de realismo y experiencia los simuladores se dividen en tres niveles: baja, mediana y alta fidelidad (Figura 3) ${ }^{35}$, los de baja solo simulan parte del organismo, los de mediana combinan la parte anatómica con programas digitales y los de alta fidelidad utilizan múltiples variables fisiológicas permitiendo el entrenamiento en técnicas avanzadas ${ }^{27}$.

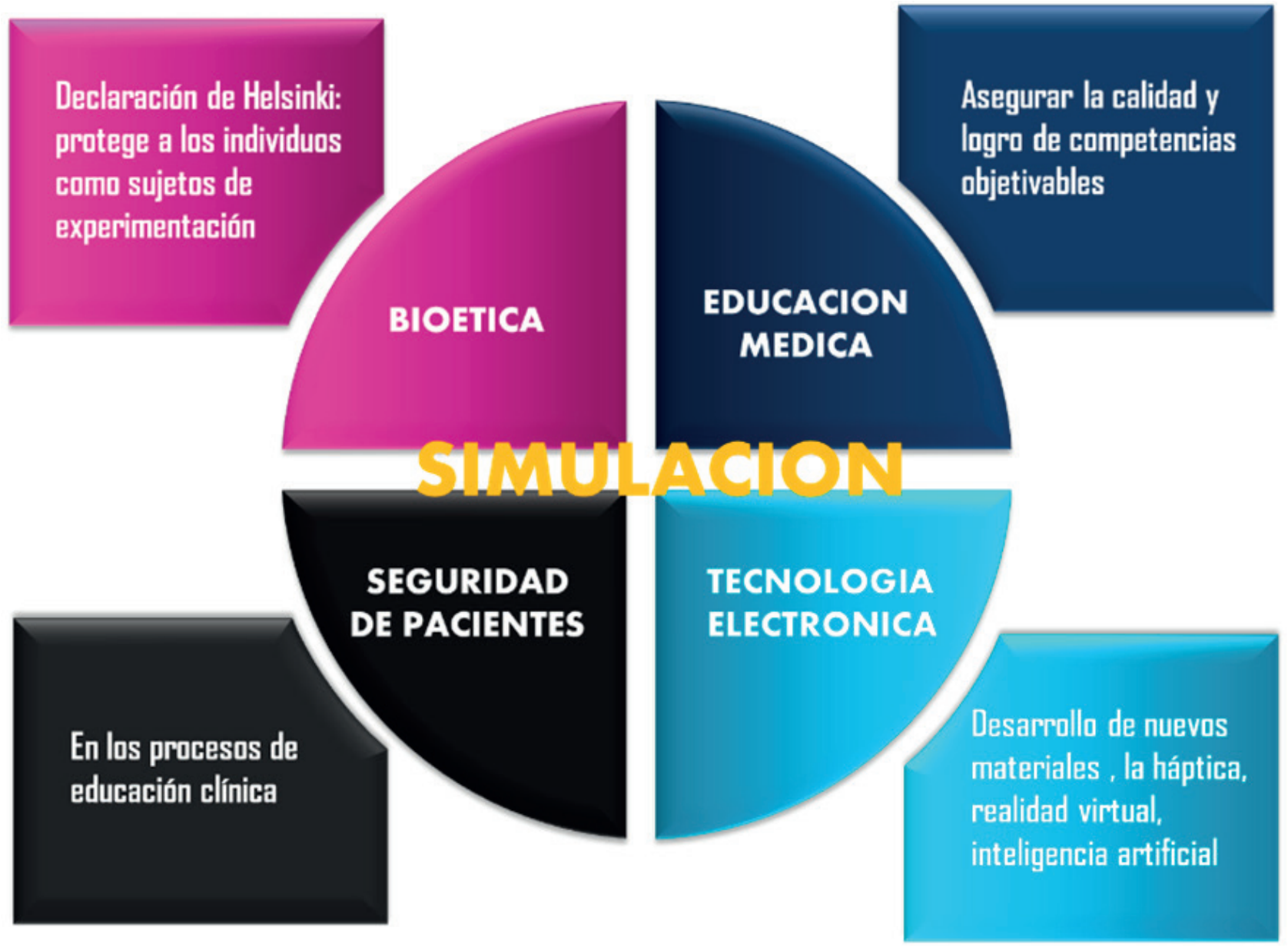

Figura 1. Fuerzas de influencia en la simulación clínica ${ }^{34}$ 


\section{NIVEL DE FIDELIDAD DE LOS SIMULADORES CLINICOS}

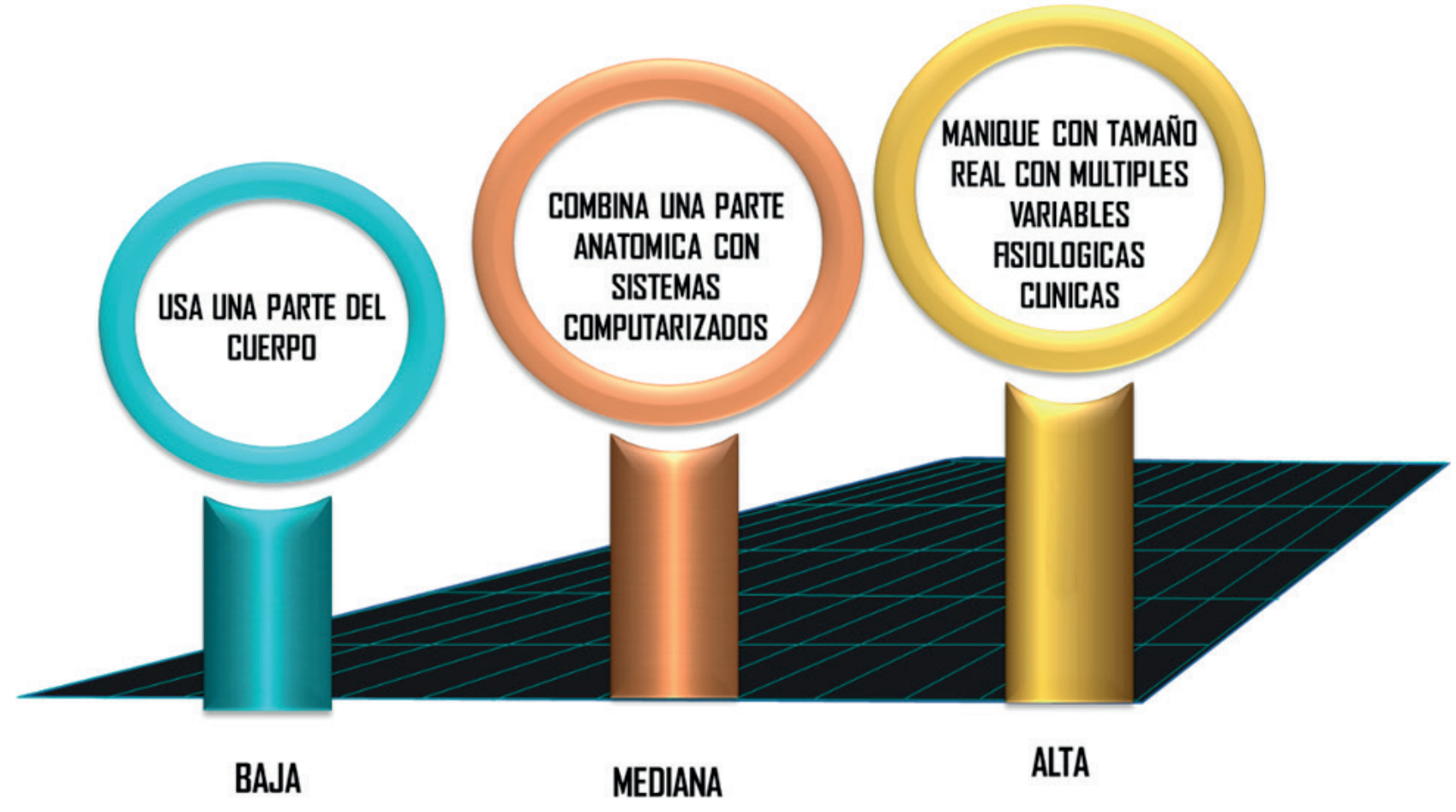

Figura 2. Clasificación de los simuladores según la fidelidad ${ }^{35}$

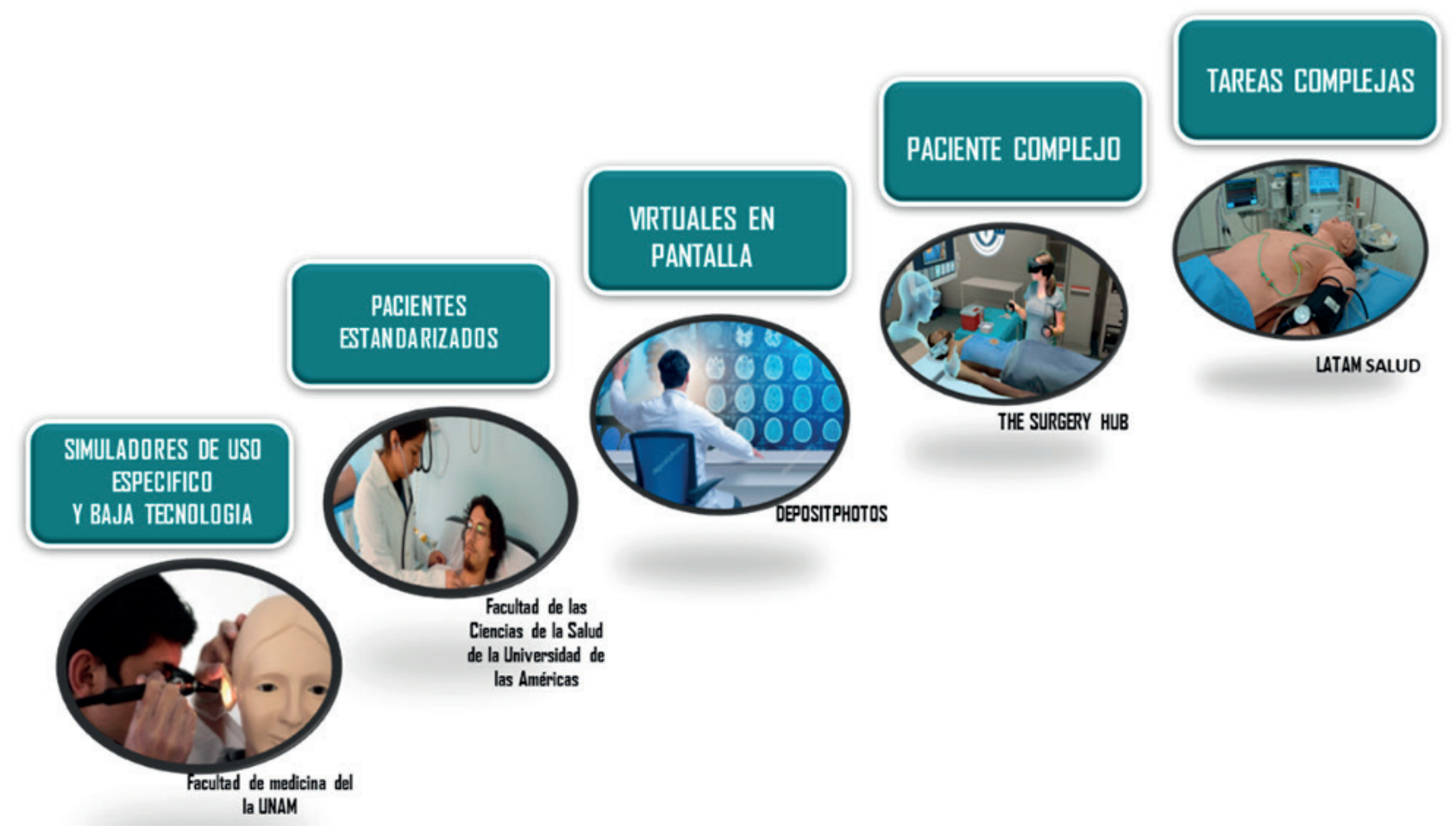

Figura 3. Clasificación de los simuladores en cinco categorías propuesta por Ziv ${ }^{36}$ 
La enseñanza en la odontología no solo requiere de una gran preparación de los docentes sino también del uso de técnicas educativas que permitan al estudiante una interacción e inmersión mediante la recreación de una parte o toda experiencia clínica, que en la actualidad la simulación cumple en gran forma este objetivo ${ }^{37}$.

Con la pandemia no es posible realizar actividades en pacientes pero los avances de las tecnologías digitales han facilitado el logro de estas competencias, acercándose cada vez con gran realismo a lo que sería una experiencia clínica, para un retorno a la presencialidad, que inicialmente será parcial. Se va a requerir la recuperación de las horas clínicas siendo la mejor alternativa el uso de los simuladores, hasta un total retorno, condición que solo se logrará mediante la disposición de las vacunas y la adecuación de los ambientes a una nueva "normalidad" Es un desafío para lo cual se deben ir tomando todas las previsiones para cuando se retorne a la universidad ${ }^{37,38}$.

Perspectivas actuales. El escenario aún es incierto para el futuro retorno a la presencialidad en las facultades de odontología, las esperanzas están centradas en el uso de la vacuna contra un virus que tiende a mutar haciéndose más contagioso, la adecuación a las plataformas virtuales aun cuando han estado disponibles desde hace algunos años, no se tomaron en cuenta, existe mucha resistencia a su uso restándole importancia, el equipamiento e infraestructura clínica no está acondicionado para enfrentar una pandemia como la del SARS-CoV-2, los protocolos de bioseguridad no tienen la rigurosidad que se requiere para controlar el contagio viral. Es necesaria una revisión y replanteamiento total de los procesos educativos y clínicos en las facultades de odontología, mientras tanto las plataformas virtuales ocuparan el mayor protagonismo en la enseñanza. Hay aspectos importantes que no deben pasar desapercibidos como es el emocional, el apoyo psicológico, la gestión del estrés condiciones que se han visto incrementadas ya sea por el temor al contagio o por el fallecimiento de familiares y que por lo tanto, no se debe descuidar ${ }^{5}$.

\section{Conclusiones}

El uso de las TICs requiere de un proceso de capacitación y actualización permanente para los docentes y estudiantes. Así también es necesario un equipamiento y conectividad segura. El uso de los simuladores es una gran alternativa para el desarrollo de las competencias procedimentales y actitudinales, siendo necesario durante su uso cumplir con las normas de bioseguridad. La simulación permite a los estudiantes el desarrollo de destrezas y habilidades mediante la repetición de los procedimientos clínicos de forma indefinida. Sin poner en riesgo la salud de los pacientes.

\section{Referencias bibliográficas}

1. Peña-Soto C. Educación en odontología en esta «nueva normalidad». Rev Cient Odontol. 2020;8(2):e014. DOI: $10.21142 / 2523-2754-0802-2020-014$.
2. Martínez CDC, Yévenes HSR. Dental caring during COVID-19 pandemic. Int J Odontostomat. 2020;14(3):288-95.

3. Instituto Internacional para la Educación superior en América Latina y el Caribe. COVID-19 y educación superior: De los efectos inmediatos al día después. UNESCO; 2020. Informe técnico:1-57.

4. Christiani JJ, Rocha MT, Valsecia M. Seguridad del Paciente en la práctica odontológica. Acta Odonto Col [Internet]. 2015 [citado el 05 de julio de 2021]; 5(2):21-32. Disponible en: http://www.revistas.unal.edu.co/index. $\mathrm{php} /$ actaodontocol.

5. Lenguas-Silva AL, Mateos-Moreno MV, García-Vicent G, Lamas-Oliveira M, Martín-Morales J, Valdepeñas-Morales J, et al. New rules for teaching in dental schools in the COVID 19 era. Int J Odontostomat [Internet]. 2021 [citado el 05 de julio de 2021]; 15(1):3642. Disponible en: https://scielo.conicyt.cl/pdf/ijodontos/v15n1/0718-381X-ijodontos-15-01-36.pdf.

6. Alzahrani SB, Alrusayes AA, Aldossary MS. Impact of Covid-19 Pandemic on dental Education, Research and Students. Int J Health Sci Res [Internet]. 2020 [citado el 05 de julio de 2021]; 10(6):207-12. Disponible en: https://www.ijhsr.org/IJHSR_Vol.10_Issue.6_ June2020/32.pdf.

7. Morón AM. La Teleodontología una Herramienta Fundamental en Tiempos de Pandemia y post COVID -19, su Utilidad en las Diferentes Especialidades Odontológicas. Int J Odontostomat [Internet]. 2021 [citado el 05 de julio de 2021]; 15(1):43-50. Disponible en: http://www.ijodontostomatology.com/wp-content/ uploads/2020/11/2021_v15n1_012.pdf.

8. Laiseca LB, Zanin A, Cid JL, Bartolomé SM. Simulation during COVID-19 pandemic in the Spanish pediatric intensive care units: New challenges in medical education. An Pediatr (Engl Ed) 2021; Epub ahead of print. DOI: 10.1016/j.anpedi.2021.06.007.

9. Hawkins RE, Welcher CM, Holmboe ES, Kirk LM, Norcini JJ, Simons KB, Skochelak SE. Implementation of competency-based medical education: are we addressing the concerns and challenges? Med Educ. 2015;49(11):1086-102. DOI: 10.1111/medu.12831.

10. Cascella M, Rajnik M, Aleem A, Dulebohn SC, Di Napoli R. Features, Evaluation, and Treatment of Coronavirus (COVID-19). In: StatPearls [Internet]. Treasure Island (FL): StatPearls Publishing; 2021.

11. Petrosillo N, Viceconte G, Ergonul O, Ippolito G, Petersen E. COVID-19, SARS and MERS: are they closely related? Clin Microbiol Infect. 2020;26(6):729-34. DOI: 10.1016/j.cmi.2020.03.026.

12. Sabino-Silva R, Jardim ACG, Siqueira WL. Coronavirus COVID-19 impacts to dentistry and potential salivary diagnosis. Clin Oral Investig. 2020;24(4):1619-21. DOI: $10.1007 / \mathrm{s} 00784-020-03248-\mathrm{x}$.

13. García-Peñalvo FJ, García-Holgado A, Vázquez-Ingelmo A, Sánchez Prieto JC. Planning, communication and active methodologies: Online assessment of the software engineering subject during the COVID-19 crisis. RIED. 2021;24(2):41-66. DOI: 10.5944/red24.2.27689. 
14. Grande-de-Prado M, García-Peñalvo FJ. Corell Almuzara A, Abella-García V. Evaluación en Educación Superior durante la pandemia de la COVID-19. Campus Virtuales [Internet]. 2021 [citado el 05 de julio de 2021]; 10(1):49-58. Disponible en: http://uajournals.com/ojs/ index.php/campusvirtuales/article/view/747/429.

15. Corell A, García Peñano FJ. COVID-19: La encerrona que transformo las universidades en virtuales. Gaceta Cultural [Internet]. 2021 [citado el 05 de julio de 2021]; 23-6. Disponible en: https://repositorio.grial.eu/bitstream/grial/2182/3/Alfredo_Fran.pdf.

16. Jiménez Guerra Y, Ruiz González M. Reflexiones sobre los desafíos que enfrenta la educación superior en tiempos de COVID-19. Econ y Desarrollo [Internet]. 2021 [citado el 05 de julio de 2021]; 165(Supl. 1),e3. Disponible en: http://scielo.sld.cu/scielo.php?script=sci_arttext\&pid=S0252-85842021000200003\&lng=es\&nr$\mathrm{m}=$ iso.

17. Hodges Ch, Moore S, Lockee B, Trust T, Bond A. The Difference Between Emergency Remote Teaching and Online Learning. Educause Review [Internet]. 2020 [citado el 05 de julio de 2021]; 1-15. Disponible en: https://er.educause.edu/articles/2020/3/the-difference-between-emergency-remote-teaching-and-online-learning.

18. Pérez-López E, Vázquez Atochero A, Cambero Rivero S. Educación a distancia en tiempos de COVID-19: Análisis desde la perspectiva de los estudiantes universitarios. RIED. 2021;24(1):331-50. DOI: 10.5944/ ried.24.1.27855.

19. Figueroa Fernández NP, Pérez Cortez G, Rojas MH, Zorrilla Martínez II, Muñoz Salcido G, Domínguez Sánchez A, et al. Comparación de indicadores del proceso docente educativo virtual en odontología, durante la epidemia de COVID-19. Congreso Internacional Estomatología 2020, 1-30 de noviembre de 2020. La Habana: Facultad de Estomatología de la Habana; 2020.

20. Núñez-Cortés JM. COVID-19 por SARS-Cov2 también ha afectado a la Educación Médica. Educ Med. 2020;21(4):261-4. DOI: 10.1016/j.edumed.2020.06.001.

21. Shah P, Paranjpe M. Effects of COVID-19 Pandemic on Dental Education in Dental Colleges of Various Universities in India. Rev OActiva Univ Católica Cuenca [Internet]. 2021 [citado el 05 de julio de 2021] ;6(1):5-8. Disponible en: file:///Users/rossemary/ Downloads/556-Texto\%20del\%20art\%C3\%ADculo-1914-1-10-20201230.pdf

22. de Obesso MM, Núñez Canal M. El modelo educativo híbrido: una respuesta necesaria de la enseńanza universitaria a partir de la COVID-19. 2021; Preprint File. DOI: 10.13140/RG.2.2.34706.89289.

23. Chuenjitwongsa S, Oliver RG, Bullock AD. Competence, competency-based education, and undergraduate dental education: a discussion paper. Eur J Dent Educ. 2018;22(1):1-8. DOI: 10.1111/eje.12213.

24. López Sánchez M, Ramos López L, Pato López O, López Álvarez S. La simulación clínica como herramienta de aprendizaje. Cir May Amb [Internet]. 2013 [citado el 05 de julio de 2021]; 18(1):25-9. Disponible en: http:// www.asecma.org/Documentos/Articulos/05_18_1_FC_ Lo\%C2\%A6\%C3\%BCpez.pdf.
25. Maran NJ, Glavin RJ. Low- to high-fidelity simulation - a continuum of medical education? Med Educ. 2003;37(1):22-8. DOI: 10.1046/j.1365-2923.37.s1.9.x.

26. Pai D. Use of simulation for undergraduate medical education. Int J Adv Med Health Res [Internet]. 2018 [citado el 05 de julio de 2021]; 5:3-6. Disponible en: https:// www.ijamhrjournal.org/text.asp?2018/5/1/3/235609.

27. Gaba DM. The future vision of simulation in health care. Qual Saf Health Care. 2004;13(Suppl 1):2-10. DOI: 10.1136/qhc.13.suppl_1.i2.

28. Ávila Juárez SA, García Barrón AM, Morales López SM. Simulación con paciente estandarizado y simuladores de baja fidelidad (PESiBaF) como primer acercamiento a un paciente en estudiantes de primer año de la carrera de médico cirujano. Educ Med. 2020;21(6):364-9. DOI: 10.1016/j.edumed.2018.10.011.

29. Fernández-Sagredo M, Barrios-Penna C, Torres-Martínez Pilar, Sáez-Espinoza R, Fonseca-Molina J. Percepción de la utilidad de los simuladores virtuales hápticos en educación odontológica por estudiantes, profesionales y académicos: estudio descriptivo observacional. FEM. 2020;23(2):89-94. DOI: 10.33588/fem.232.1045.

30. Suasnabar-Pacheco L, Campos-Mancero OV, Rivera-Guerrero CP, Zumba-Macay R, Escudero-Doltz W. Una mirada de las tecnologías de información y la comunicación en odontología. Dom Cienc. 2019;5(2):497522. DOI: $10.23857 /$ dc.v5i2.917.

31. García-Peñalvo FJ, Corell A. La CoVId-19: ¿enzima de la transformación digital de la docencia o reflejo de una crisis metodológica y competencial en la educación superior? Campus Virtuales [Internet]. 2020 [citado el 05 de julio de 2021]; 9(2), 83-98. Disponible en: http://hdl. handle.net/10366/144140.

32. Tisi-Lanchares JP. La simulación clínica posterior a la pandemia COVID-19. Odovtos Int J Dent Sci. 2020;22(3):16-8. DOI: 10.15517/ijds.2020.42166.

33. Pérez LD. Estudio ergonómico en odontólogo durante tratamientos de endodoncia. EID [Internet]. 2020 [citado el 05 de julio de 2021]; 2(1):168-81. Disponible en: https://revistasacademicas.udec.cl/index.php/Ergonomia_Investigacion/article/view/1987.

34. Corvetto M, Bravo MP, Montańa R, Utili F, Escudero E, Boza C, et al. Simulación en educación médica: una sinopsis. Rev Med Chile. 2013;141(1):70-9. DOI: 10.4067/S0034-98872013000100010.

35. Dávila-Cervantes A. Simulación en Educación Médica. Inv Edu Med. 2014;3(10):100-5. DOI: 10.1016/S20075057(14)72733-4.

36. Ziv A, Wolpe PR, Small SD, Glick S. Simulation-based medical education: an ethical imperative. Simul Healthc. 2006;1(4):252-6. DOI: 10.1097/01. SIH.0000242724.08501.63.

37. Munshi F, Lababidi H, Alyousef S. Low-versus high-fidelity simulations in teaching and assessing clinical skills. J Taibah Univ Med Sci. 2015;10(1):12-5. DOI: 10.1016/j.jtumed.2015.01.008.

38. Duran-Ojeda G. Educación en odontología para las asignaturas de simulación preclínica en tiempos de Pandemia por COVID-19. Odovtos Int J Dental Sci. 2020;22(2):11-3. DOI: 10.15517/ijds.2020.41496. 